\title{
ARTICLE
}

\section{Wastes after distillation of Helichrysum italicum - biological active compounds and free radical scavenging activity}

\author{
Milena Nikolova ${ }^{1 *}$ Ana Dobreva² Strahil Berkov¹ \\ ${ }^{1}$ Institute of Biodiversity and Ecosystem Research, Bulgarian Academy of Sciences, Sofia, Bulgaria \\ ${ }^{2}$ Institute of Roses, Essential and Medicinal Cultures, Kazanluk, Bulgaria
}

\begin{abstract}
Distillation wastewater, by-products from steam and water distillation as well as raw material used as control of flower heads of Helichrysum italicum were comparative analyzed for content of the biologically active compounds by GC/MS. Acetone exudates, methanol extracts and ethyl acetate fractions obtained after alkaline hydrolyze of the studied materials were received. The three types of extraction products as well as the distillation wastewater were examined for free radical scavenging activity by DPPH assay. Phenol, fatty- and organic acids, sterols, triterpenes, sugars and sugar alcohols were identified. Succinic acid and myo-inositol were identified as main components of distillation wastewater. Hydroxycinnamic acid, caffeic acid and 4(p)-hydroxybenzoic acid were dominant compounds of the ethyl acetate fractions. Triterpenes and fatty acids, sterols and flavonoids are among the main biologically active substances in the methanolic extracts and acetone exudates. The ethyl acetate fractions were found to possess the highest free radical scavenging activity $\left(\mathrm{IC}_{50}<50 \mu \mathrm{g} / \mathrm{mL}\right)$. Significant differences in the activity between wastes and raw materials were not found. The results showed that the waste products after distillation of $\mathrm{H}$. italicum contain important biologically active substances and the extracts with high antioxidant activity can be obtained from them. Acta Biol Szeged 64(2):233-237 (2020)
\end{abstract}

\author{
KEY WORDS \\ GC/MS \\ flavonoids \\ phenolic acids \\ triterpenes \\ sterols
}

\section{Introduction}

Helichrysum italicum (Roth) G. Don. is an endemic Mediterranean plant, widely used in medicine and perfumery because of anti-inflammatory, analgesic, antibacterial, antistress, anti-depressant sedative properties of its essential oil (Saint-Lary et al. 2018; Staver et al. 2018; Ninčević et al. 2019). An inescapable pressure on this wild resource in the last decade resulted in a shift from wild harvest to cultivation. Its plantation farming experienced a huge expansion in Eastern European Mediterranean countries. In Bulgaria the cultivation and processing of Helichrysum has been done for several years. As a result of the intensive use of the species for extraction of essential oil a large amount of agro-industrial residues and by-products remain. The content of bioactive compounds in these wastes and their potential for application has not been investigated so far. Utilization of these wastes would be important for the conservation of natural resources and also from the point of view for the global concept of agro-waste usage (Otles et al. 2015; Saha et al. 2019). Two major types of waste are generated: wastewater and solid waste biomass. The main approaches to biomass conversion are combustion, composting, conventional and advanced extraction process, chemical and/or enzymatic reactions and development of bioprocess (Kalra et al. 2002; Santana-Méridas et al. 2012; Slavov et al. 2017). Two common types of substances are mostly recovered as by-products (besides the aroma compounds): polyphenols and polysaccharides. Most of the applications of these chemical-based products have been addressed to exploit their antioxidant, cosmeceutical and/or pharmacological properties (Fierascu et al. 2019; Makris et al. 2019; Matos et al. 2019; Pandey et al. 2010; Slavov et al. 2019). The residual distillation water of some aromatic plant may influence monoterpene synthesis and accumulation in plants and hence may be used for targeted modification of its essential oil composition (Zheljazkov and Astatkie 2012; Zheljazkov et al. 2010). Another investigation reveals potential to recovery of polyphenols (Rusanov et al. 2014).

The purpose of the present study was to determine the bioactive compounds composition and the free radical scavenging activity of various waste products from the distillation of $H$. italicum, with a view to guidelines for their valorization and application. 


\section{Materials and Methods}

\section{Plant material}

Helichrysum italicum was from the plantation in Institute for Roses and Essential and Medicinal Cultures, Kazanlak, Bulgaria. The plants originated from Bosnia. They were two years old at the time of the investigation. The biomass was obtained after vapor and water distillation in the semi-industrial installation with $100 \mathrm{dm}^{3}$ vessel. The wastewater was collected after water distillation.

\section{Extractions of plant materials}

Acetone exudates were prepared from air-dried, not grounded aerial parts of the samples (raw material and waste from distillation) by rinsed with acetone for $5 \mathrm{~min}$ to dissolve the material accumulated on the surfaces. Methanolic extracts were prepared from air-dried, ground plant parts of studied samples by classical maceration with methanol for $24 \mathrm{~h}$. Ethyl acetate fractions were obtained by alkaline hydrolyze of the plant material of the studied samples by $2 \mathrm{M} \mathrm{NaOH}$, for $4 \mathrm{~h}$ at room temperature. After acidification to $\mathrm{pH} \mathrm{1-2} \mathrm{with} \mathrm{cc.} \mathrm{HCl}$, the phenolic compounds were extracted with EtOAc two times and after that evaporated to obtain fraction rich on alkaline hydrolysable phenolic acids. Distillation wastewater was evaporated to dryness. The obtained extractions, fractions and dry residue from the distillation water were silylated with $50 \mu \mathrm{L}$ of N,O-bis-(trimethylsilyl)trifluoro-acetamide (BSTFA) in $50 \mu \mathrm{L}$ of pyridine for $2 \mathrm{~h}$ at $50{ }^{\circ} \mathrm{C}$.

\section{Gas chromatography mass spectrometry (GC-MS) analy- sis.}

The GC-MS spectra were recorded on a Thermo Scientific Focus gas chromatograph coupled with Thermo Scientific dual stage quadrupole (DSQ) mass detector operating in electron ionization (EI) mode at $70 \mathrm{eV}$. A DB-5MS column (30 m x $0.25 \mathrm{~mm} \times 0.25 \mu \mathrm{m})$ was used. Chromatographic conditions were described by Nikolova et al. (2016). The metabolites were identified as TMSi derivatives comparing their mass spectra and Kovats Indexes (RI) with those of an on-line available plant specific database (Golm Metabolome Database). The amounts of the metabolites $(\mu \mathrm{g} / \mathrm{mL})$ are expressed relative to the internal standard (3,4 dichloro-4-hidroxybenzoic acid) using the calculated areas for both components.

\section{Free radical scavenging activity}

The effect of methanolic extracts, acetone exudates and ethyl acetate fractions as well as distillation wastewater on DPPH radicals was estimated according to Stanojević et al. (2009). The results were calculated by GraphPad Prism ver. 3.00. All experiments were carried out in triplicate.

\section{Results}

Methanolic extracts, acetone exudates and ethyl acetate fractions were obtained in search for biologically active substances in the waste products from the distillation of the flower heads of $H$. italicum. Thirty-two compounds were identified. Acetone exudates contain lipophilic compounds accumulated on the surface areas of the plant material whereas methanolic extracts contain polar and lipophilic compounds. The identified compounds are presented at Table 1 . In the acetone exudates the main components were identified as triterpene acids $(11,12)$. Stigmasterol (8), $\beta$-sitosterol (9) and $\beta$-amyrin (10) were established also. Flavonoid aglycones - quercetin (24) and methyl derivatives of apigenin $(22,23)$ and luteolin $(25)$ were detected. A variety of phenolic acids - caffeic (32), 4(p)-hydroxybenzoic (26), vanillic, (27) protocatechuic (28), hydroxycinnamic (30) were found. Fatty acids and alcohols were also determined. Chlorogenic (33) and quinic (29) acids, kaempferol 4-methyl ether (21) and tocopherol (6) were found only in the acetone exudate of raw material.

In the methanolic extracts monosaccharides - fructose (18), glycose (19) and sugar derivatives - myo-inositol (20) as well as triterpene acids were found in the significant amounts. Free phenolic acids, fatty- and organic acids as well as flavonoids were also found.

Ethyl acetate fractions comprise mainly alkaline hydrolysable phenolic acids. Caffeic (32) and hydroxycinnamic (30) acids were determined as main phenolic acids. Protocatechuic acid (28) was also abundant in the fraction of untreated flower heads moreover, gallic acid (31) was found only in this sample. The detailed information is presented at Table 2 .

In the distillation wastewater the main component was identified as myo-inositol (sugar alcohol) (20) around

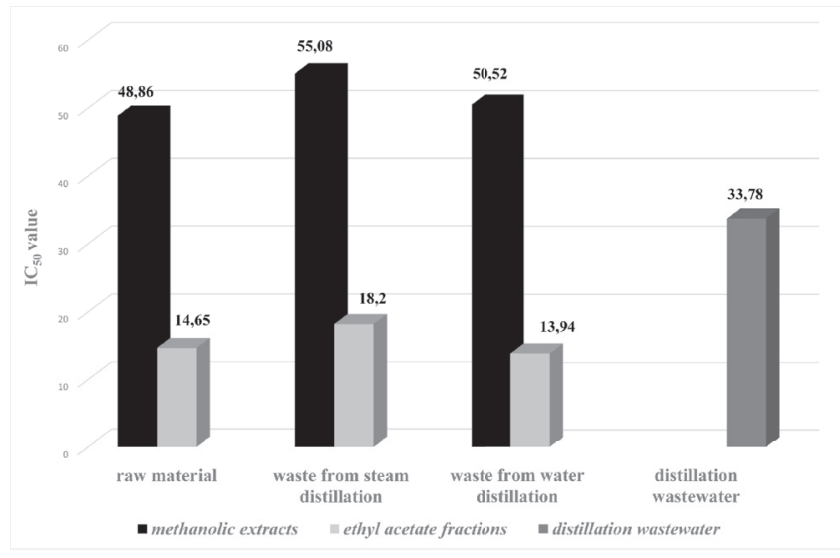

Figure 1. Free radical scavenging activity of the wastes after distillation and raw material of $H$. italicum. 
Table 1. Compounds identified of acetone exudates and methanolic extracts of the wastes after distillation and raw material of $H$. italicum.

\begin{tabular}{|c|c|c|c|c|c|c|c|c|}
\hline \multirow[t]{2}{*}{ Compounds } & \multirow[t]{2}{*}{$\mathbf{R I}$} & \multicolumn{3}{|c|}{ Acetone exudates* } & \multicolumn{3}{|c|}{ Methanolic extracts* } & \multirow[t]{2}{*}{ DWW } \\
\hline & & RM & SD & WD & RM & SD & WD & \\
\hline \multicolumn{9}{|l|}{ Fatty alcohols and acids } \\
\hline Tetradecanol (1) & 1757 & 36.4 & 8.1 & 0.4 & 0.1 & & 2.8 & \\
\hline Hexadecanol (2) & 1955 & 8.2 & 7.4 & 5.95 & 14.5 & 5.5 & 13.4 & \\
\hline Hexadecanoic acid (3) & 2041 & 292.7 & 28.5 & 28.5 & 519.3 & 686.2 & 741.8 & \\
\hline Octadecanoic acid (4) & 2124 & 222 & 10.4 & 62 & 56.8 & 3.6 & 4.3 & \\
\hline Hexacosanol (5) & 2938 & 99.3 & 0.1 & 0.8 & 176.2 & 0.1 & 73.4 & \\
\hline \multicolumn{9}{|l|}{ Sterols and triterpenes } \\
\hline Tocopherol (6) & 3122 & 6.9 & & & 12.2 & & & \\
\hline Campesterol (7) & 3265 & & 16.4 & 9 & 0.1 & & 1.5 & \\
\hline Stigmasterol (8) & 3319 & 33.25 & 61.05 & 47.6 & 37.8 & 2.4 & 12.7 & \\
\hline$\beta$-Sitosterol (9) & 3335 & 16 & 138.4 & 27.1 & 33 & 8.2 & 13.7 & \\
\hline$\beta$-Amyrin (10) & 3415 & 9.13 & 8.1 & 9.1 & 0.2 & 0.2 & & \\
\hline Triterpene acid 1 (11) & 3733 & 46.45 & 122 & 55.95 & 153.7 & 77.2 & 580 & \\
\hline Triterpene acid 2 (12) & 3778 & 156.2 & 386 & 122.7 & 463.4 & 1268.5 & 5117.9 & \\
\hline \multicolumn{9}{|c|}{ Organic acids, sugars and sugar derivatives } \\
\hline Phosphoric acid (13) & 1120 & 14.9 & 0.2 & 2.2 & 33 & 5.4 & 11.4 & 0.2 \\
\hline Succinic acid (14) & 1310 & 14.9 & 0.9 & 6.25 & 26.5 & 23.6 & 67.3 & 26.9 \\
\hline Malic acid (15) & 1488 & 16.1 & 1.7 & 2.2 & 28.5 & 43.9 & 184.1 & \\
\hline Meso-erythritol (16) & 1493 & & & & 31.3 & 5.3 & 4.2 & 0.5 \\
\hline Pyroglutamic acid (17) & 1512 & & & 9.75 & 10.1 & & 31.8 & 1 \\
\hline Fructose (18) & 1805 & 88.1 & 8.65 & 8.7 & 410.3 & 326.2 & 230.7 & \\
\hline Glucose (19) & 1882 & 176.4 & 3.85 & 3.8 & 157.2 & 227.4 & 195.6 & \\
\hline Myo-Inositol (20) & 2093 & 6.6 & 10.6 & 1.4 & 42.4 & 497.4 & 749.6 & 66.5 \\
\hline \multicolumn{9}{|l|}{ Flavonoid aglycones } \\
\hline Kaempferol 4-Me (21) & 3001 & 0.45 & & & 0.08 & & & \\
\hline Apigenin 4ロ-Me (22) & 3040 & 5.2 & 2.8 & 2.05 & & 0.4 & 1.5 & \\
\hline Scutellarein 6,4ロ-Me (23) & 3108 & 6.9 & 7.8 & 3.7 & & 0.9 & & \\
\hline Quercetin (24) & 3192 & 0.2 & & 0.5 & 0.6 & & & \\
\hline Luteolin 7,4ロ-Me (25) & 3240 & 1 & 0.5 & 1.4 & 0.05 & 0.6 & 2.2 & \\
\hline \multicolumn{9}{|l|}{ Free phenolic acids } \\
\hline 4(p)-hydroxybenzoic acid (26) & 1635 & 2.75 & 4.3 & 3.4 & 15 & 6.4 & 2.2 & \\
\hline Vanillic acid (27) & 1776 & 0.5 & 1.55 & 4.55 & 1.4 & 10.9 & 11.10 & 0.3 \\
\hline Protocatechuic acid (28) & 1813 & 0.1 & 3.95 & 4.3 & 34.3 & 2.5 & 27.2 & \\
\hline Quinic acid (29) & 1863 & 38 & 0.6 & & 98.1 & 170.2 & 30.4 & \\
\hline Hydroxycinnamic acid (30) & 1932 & & 2.6 & 1.5 & 5.8 & 0.9 & 4 & \\
\hline Gallic acid (31) & 1960 & & & & 1.6 & 2.7 & & \\
\hline Caffeic acid (32) & 2131 & 13.65 & 1.4 & 0.5 & 9.1 & 5.6 & 19.6 & \\
\hline Chlorogenic acid (33) & 3110 & 3.8 & & & 16.2 & 1.4 & 28.2 & \\
\hline
\end{tabular}

RM - raw material; SD - waste after steam distillation; WD - waste after water distillation; DWW- distillation waste water; Me - methyl ether * data were expressed for each analyte relative to the internal standard (3,4 dichloro-4-hidroxybenzoic acid) using the calculated areas for both components

$66 \%$ of all identified compounds. Succinic acid (14) was the second component presented also in large amount. The results are presented at Table 1.

Methanolic extracts, acetone exudates and ethyl acetate fractions as well as distillation wastewater were evaluated for free radical scavenging activity against DPPH radicals. The results are presented as $\mathrm{IC}_{50}$ values - extract concentration providing 50\% inhibition of the DPPH solution (Fig.1). With exception of acetone exudates, the methanolic extracts, ethyl acetate fractions and distillation wastewater showed significant activity with $\mathrm{IC}_{50}$ values less than $50 \mu \mathrm{g} \mathrm{mL}^{-1}$. The ethyl acetate fractions displayed 
Table 2. Phenolic acids identified of ethyl acetate fractions of the wastes after distillation and raw of $\mathrm{H}$. italicum.

\begin{tabular}{lllll}
\hline Phenolic acids & RI & RM & SD & WD \\
\hline Salicylic acid (34) & 1210 & 1.9 & 0.7 & \\
Cinnamic acid trans (35) & 1376 & 2.6 & & \\
4(p)-hydroxybenzoic acid (26) & 1635 & 39.2 & 22.1 & 4.2 \\
Vanillic acid (27) & 1776 & 20.4 & 7.4 & 2.2 \\
Hydroxycinnamic acid cis (36) & 1789 & 43.7 & 5.7 & 2 \\
Protocatechuic acid (28) & 1813 & 173.9 & 38.4 & 6.6 \\
Syringic acid (36) & 1884 & 10.8 & 0.9 & \\
Hydroxycinnamic acid trans (30) & 1948 & 168.5 & 110.3 & 85 \\
Gallic acid (31) & 1960 & 5.7 & & \\
Ferulic acid trans (37) & 2103 & 7.4 & 7.7 & 1 \\
Caffeic acid trans (32) & 2142 & 260.8 & 881.5 & 159.5 \\
\hline
\end{tabular}

RM: raw material; SD: waste after steam distillation; WD: waste after water distillation.

* Data were expressed for each analyte relative to the internal standard (3,4 dichloro-4-hidroxybenzoic acid) using the calculated areas for both components

the most significant activity whereas the acetone exudates the lowest upper $200 \mu \mathrm{g} \mathrm{mL}^{-1}$. Significant difference in the antiradical properties between extracts and fractions of different waste products was not found.

\section{Discussion}

The results presented show that the waste products from the distillation of essential oil of H. italicum contain important bioactive compounds. Triterpenes and sterols are bioactive substances that have been shown to possess anti-inflammatory, antimicrobial, antiallergic, antiviral, hepatoprotective, cytotoxic and other important biological activities (Holanda et al. 2008; Sultana and Ata 2008; Vázquez et al. 2012). The presence of triterpenes in H. italicum has already been reported in the literature (Mezzetti et al. 1970; Nostro et al. 2000; Guinoiseau et al. 2013). The occurrence of triterpene acids in significant amounts in the extracts from by-products after steam and water distillation was found in the present study. Significant free radical scavenging activity has been reported for $H$. italicum (Molnar et al. 2017) that is consistent with received data of DPPH assay in the present work. Phenolic acids have been determined as main contributors of antioxidant properties (Sato et al. 2011). A variety of this class compounds were established in the methanolic extracts and ethyl acetate fractions of the waste products and raw materials. The high antiradical activity of the extracts and fractions is suggested to be due precisely to the high content of phenolic acids in them. Myo-inositol and succinic acid were established as dominant components in the distillation wastewater. Antitumor properties, beneficial effect on diseases such as diabetes, obesity and neurodegenerative disorders have been reported for myo-inositol and its derivatives (Bizzarri et al. 2016; Chhetri, 2019). Antibacterial action and use in the treatment of acne has been reported for succinic acid (Wang et al. 2014). Later research outlined succinic acid as a promising compound for use in cosmetics (Theunissen and Courbes 2018). These data indicate that distillation wastewater could find application in cosmetic products.

\section{Conclusion}

The results presented here indicate that the waste products from the distillation of $H$. italicum contain important bioactive compounds - triterpene acids, sterols, phenolic acids, sugar alcohols - substances with important biological activities. These data outline the possibility from the waste materials of $H$. italicum to be recovered fractions or pure compounds with valuable properties. Finding application of these waste products will allow a fuller use of the plant material of the species.

\section{References}

Akaberi M, Sahebkar A, Azizi N, Emami SA (2019) Everlasting flowers: phytochemistry and pharmacology of the genus Helichrysum. Ind Crops Prod 138:111471.

Bizzarri M, Dinicola S, Bevilacqua A, Cucina A (2016) Broad spectrum anticancer activity of myo-inositol and inositol hexakisphosphate. Int J Endocrinol 2016:5616807.

Chhetri DR (2019) Myo-inositol and its derivatives: Their emerging role in the treatment of human diseases. Front Pharmacol 10:11-72.

Facino M, Carini M, Franzoi L, Pirola O, Bosisio E (1990) Phytochemical characterization and radical scavenger activity of flavonoids from Helichrysum italicum G. Don (Compositae). Pharmacol Res 22:709-720.

Fierascu R, Fierascu I, Avramescu S, Sieniawska E (2019) Recovery of natural antioxidants from agro-industrial side streams through advanced extraction techniques. Molecules 24(23):4212.

Guinoiseau E, Lorenzi V, Luciani A, Muselli A, Costa JP, Casanova JG, Berti L (2013) Biological properties and resistance reversal effect of Helichrysum italicum (Roth) G. Don. In Méndez-Vilas A, Ed., Formatex Research Center, Spain, 1073-1080.

Holanda Pinto SA, Pinto LM, Cunha GM, Chaves MH, Santos FA, Rao VS (2008) Anti-inflammatory effect of alpha, beta-amyrin, a pentacyclic triterpene from 
Protium heptaphyllum in rat model of acute periodontitis. Inflammopharmacology 16(1):48-52.

Kalra A, Kumar S, Katiyar N, Bahl JR, Bansal RP, Chauhan HS, Prasad A, Pandey R, Dhawan OP, Krishna A, Srivastava R (2002) Method for the faster multiplication of earthworms, and production of vermicompost from the distillation waste of industrial aromatic crops. Patent No: US 6,488,733 B2.

Makris D, Sahin S (2019) Polyphenolic antioxidants from agri-food waste biomass. Antioxidants 8(12):624.

Matos MS, Romero-Díez R, Álvarez A, Bronze MR, Rodríguez-Rojo S, Mato RB, Cocero MJ, Matias AA (2019) Polyphenol-rich extracts obtained from winemaking waste streams as natural ingredients with cosmeceutical potential. Antioxidants 8(9):355.

Mezzetti T, Orzalesi G, Rossi C, Bellavita V (1970) A new triterpenoid lactone, alpha-amyrin and uvaol from $\mathrm{He}$ lichrysum italicum. Planta Med 18(4):326-331.

Molnar M, Jerković I, Suknović D, Bilić Rajs B, Aladić K, Šubarić D, Jokić S (2017) Screening of six medicinal plant extracts obtained by two conventional methods and supercritical $\mathrm{CO}_{2}$ extraction targeted on coumarin content, 2,2-diphenyl-1-picrylhydrazyl radical scavenging capacity and total phenols content. Molecules 22:348.

Nikolova M, Aneva I, Berkov S (2016) GC-MS metabolic profiling and free radical scavenging activity of Micromeria dalmatica. Biol Nyssana 7(2):159-165.

Ninčević T, Grdiša M, Šatović Z, Jug-Dujaković M (2019) Helichrysum italicum (Roth) G. Don: Taxonomy, biological activity, biochemical and genetic diversity. Ind Crops Prod 138:111487.

Nostro A, Germanò MP, D'angelo V, Marino A, Cannatelli MA (2000) Extraction methods and bioautography for evaluation of medicinal plant antimicrobial activity. Lett Appl Microbiol 30(5):379-384.

Otles S, Despoudi S, Bucatariu C, Kartal C (2015) Food waste management, valorization, and sustainability in the food industry. In Galanakis CM, Ed., Academic Press, Cambridge, 3-23.

Pandey R, Kalra A (2010) Inhibitory effects of vermicompost produced from agro-waste of medicinal and aromatic plants on egg hatching in Meloidogyne incognita (Kofoid and White) chitwood. Curr Sci 98(6):833-835.

Rusanov K, Garo E, Rusanova M, Fertig O, Hamburger M, Atanassov I, Butterweck V (2014) Recovery of polyphenols from rose oil distillation wastewater using adsorption resins - a pilot study. Planta Med 80:1657-1664.

Theunissen L, Courbes F (2018) Succinic acid: a promising multi-functional ingredient for cosmetic and personalcare applications. H\&PC Today 13(2):42-44.
Saha A, Basak BB (2020) Scope of value addition and utilization of residual biomass from medicinal and aromatic plants. Ind Crops Prod 145:111979.

Saint-Lary L, Minagloub FH, Escrivac C, Beylsa AS, Badie F (2018) Helichrysum italicum D.C. essential oil from Balkans. Perfum Flavor 43:52-66.

Santana-Méridas O, González-Coloma A, Sánchez-Vioque R (2012) Agricultural residues as a source of bioactive natural products. Phytochem Rev 11(4):447-466.

Slavov A, Vasileva I, Stefanov L, Stoyanova A (2017) Valorization of wastes from the rose oil industry. Rev Environ Sci Biotechnol 16:309-325.

Slavov A, Yantcheva N, Vasileva I (2019) Chamomile wastes (Matricaria chamomilla): New source of polysaccharides. Waste Biomass Valor 10(9):2583-2594.

Staver M, Gobin I, Ratkaj I, Petrovic M, Vulinovic A, Dinarina-Sablic M, Broznic D (2018) In vitro antiproliferative and antimicrobial activity of the essential oil from the flowers and leaves of Helichrysum italicum (Roth) G. Don growing in central Dalmatia (Croatia).J Essent Oil-Bear Plants 21(1):77-91.

Stanojević L, Stanković M, Nikolić V, Nikolić L, Ristić D, Canadanovic-Brunet J, Tumbas V (2009) Antioxidant activity and total phenolic and flavonoid contents of Hieracium pilosella L. extracts. Sensors 9:5702-5714.

Sultana N, Ata A (2008) Oleanolic acid and related derivatives as medicinally important compounds. J Enzyme Inhib Med Chem 23:739-756.

Zeljković S, Šolić ME, Maksimović M (2015) Volatiles of Helichrysum italicum (Roth) G. Don from Croatia. Nat Prod Res 29(19):1874-1877.

Zheljazkov V, Astatkie T (2012) Distillation waste water can modify peppermint (Mentha $\times$ piperita L.) oil composition. Ind Crops Prod 36(1):420-426.

Zheljazkov VD, Astatkie T, Horgan T, Rogers S (2010) Effect of plant hormones and distillation water on mints. HortScience 45(9):1338-1340.

Vázquez LH, Palazon J, Navarro-Ocaña A (2012) The pentacyclic triterpenes $\alpha-\beta$-amyrins: a review of sources and biological activities. In Venketeshwer R, Ed., Phytochemicals - A Global Perspective of Their Role in Nutrition and Health. IntechOpen, Rijeka, Croatia.

Wang Y, Kuo S, Shu M, Yu J, Huang S, Dai A, Two A, Gallo RL, Huang CM (2014) Staphylococcus epidermidis in the human skin microbiome mediates fermentation to inhibit the growth of Propionibacterium acnes: implications of probiotics in acne vulgaris. Appl Microbiol Biotechnol 98(1):411-424. 
